\title{
Commande multivariable du moteur asynchrone triphasé à cage par variation de fréquence
}

Michel Pierre RABenarivo RAZAFIndRaIBE ${ }^{*}$ et Paul Auguste RANDRIAMItANTSOA²

'Université d'Antananarivo, Ecole supérieure Polytechnique, Département Génie Electrique, B.P 1500, (101) Antananarivo, Madagascar

2 Université d'Antananarivo, Ecole supérieure Polytechnique, Département

Télécommunications, B.P 1500, (101) Antananarivo, Madagascar.

\footnotetext{
*Correspondance,courriel : rabenarivo.michel@yahoo.fr
}

\section{Résumé}

La commande du moteur asynchrone triphasé à cage alimenté par des convertisseurs est traitée comme celle d'un système multivariable. L'étude du comportement du moteur a été réalisée en simulation, avec différentes valeurs de la fréquence d'alimentation, pour justifier le choix de l'onduleur. Le programme de simulation établi permet d'avoir toutes les caractéristiques en introduisant les paramètres de construction du moteur. Les résultats obtenus sont utilisés pour faire l'analyse et la synthèse du système à l'aide du logiciel MATLAB.

Mots-clés : commande, système multivariable, variation de fréquence, asynchrone.

\section{Abstract}

Three phase squirrel cage asynchronous motor MIMO control by frequency variation

Three phase squirrel cage asynchronous motor control fed by rectifier and voltage inverter is treated as a MIMO system. Motor behaviour has been simulated using different values of stator supply frequency, to justify the choice of voltage inverter. Established simulation program allows to know all characteristics by inserting the parameters of the motor. The obtain result is used for the analysis and the synthesis of the system by MATLAB software.

Keywords : control, MIMO system, frequency variation, asynchronous. 


\section{Introduction}

Le besoin continuel d'augmenter les performances des procédés technologiques a permis aux actionneurs électriques, tels que les moteurs, de se trouver à l'un des premiers rangs de l'industrie. La conduite du procédé dépend du type de moteur utilisé et surtout du système de commande adopté. Les moteurs à courant continu et les moteurs synchrones ont trouvé de larges applications dans le domaine des systèmes automatisés.

Néanmoins, ces types de moteur présentent quelques inconvénients tels que leur coût relativement élevé, leur encombrement, leur entretien périodique fastidieux, la restriction de leur domaine d'installation. On peut dire que les moteurs électriques à champ tournant, apparus vers la fin du $19^{\text {ème }}$ siècle, font partie des outils de base de la civilisation moderne grâce aux énormes progrès de l'électronique de puissance. Ils occupent un vaste espace dans presque toutes les formes de transformations d'énergie. Cette omniprésence est due notamment à leur coût modéré, à leur robustesse notoire, ainsi qu'à leurs multiples qualités pour ne citer que le peu d'entretien qu'ils nécessitent et leur durée de vie relativement longue. II On a logiquement vu apparaître les actionneurs électriques, de façon massive, dans les transports guidés tels que les trains, les métros ainsi que les voitures électriques. La première génération de trains à grande vitesse a utilisé des moteurs à courant continu, la deuxième a eu recours à des moteurs synchrones, et la troisième s'est emparé des moteurs asynchrones à contrôle vectoriel associés à des onduleurs à G.T.0 ॥[1].

La représentation d'état est le seul outil qui permet de faire l'analyse et la synthèse des systèmes multivariables. On développe, dans cette étude, les équations fondamentales de simulation. La simulation en régime dynamique donne la représentation d'état du moteur. Le choix du type de redresseur, le calcul des éléments du filtre, et la modélisation de l'onduleur donnent la possibilité de former la matrice système du processus. La matrice système permet alors d'obtenir par simulation les courbes des valeurs singulières, les courbes de gain, de faire l'analyse de la robustesse en stabilité et de la robustesse en performance du système. 


\section{Matériel et méthodes}

\section{2-1. 1Modélisation vectorielle du moteur asynchrone triphasé à cage}

Toutes les relations qui suivent sont établies avec la notation habituelle :

$\checkmark$

$s$ : vecteur tournant tension statorique

$f_{s}$ : fréquence statorique

$I_{s}, I_{r}$ : vecteurs tournants courants statorique et rotorique

$\Phi_{s}, \Phi_{r}$ : vecteurs tournants flux statorique et rotorique

$T$

${ }^{T}$ em : couple électromagnétique

$R$

$s$ : résistance statorique

$R$

$r$ : résistance rotorique

$L_{s}:$ inductance cyclique statorique

$L_{r}$ : inductance cyclique rotorique

$L$

$s r$ : inductance mutuelle cyclique

$\sigma$ : coefficient de dispersion de Blondel

$\omega$

$s$ : pulsation statorique

$\omega_{r}:$ pulsation rotorique

$\Omega$ : vitesse de rotation mécanique

$\tau_{s}$ : constante de temps statorique

$\tau_{r}$ : constante de temps rotorique

La représentation vectorielle d'une grandeur triphasée peut s'exprimer dans différents référentiels (S), (R), et (T): 


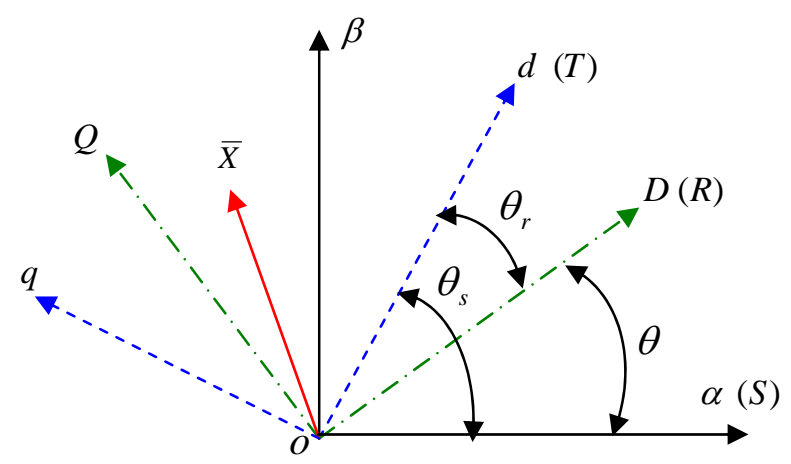

Figure 1 : Position des référentie/s

L'utilisation de la représentation complexe permet de simplifier l'écriture. Ainsi la tension statorique exprimée dans le référentiel ( $S$ )lié au stator :

$\bar{V}_{s(s)}=R_{s} \bar{I}_{s(s)}+\frac{d \bar{\Phi}_{s}(s)}{d t}$

la tension rotorique exprimée dans $(R$ )lié au rotor :

$\bar{V}_{r(R)}=\overline{0}=R_{r} \bar{I}_{r(R)}+\frac{d \bar{\Phi}_{r(R)}}{d t}$

Les expressions des flux :

$$
\begin{aligned}
& \bar{\Phi}_{s(S)}=L_{s} \bar{I}_{s(s)}+L_{s r} e^{j \theta} \bar{I}_{r(R)} \\
& \bar{\Phi}_{r(R)}=L_{r} \bar{I}_{r(R)}+L_{s r} e^{-j \theta} \bar{I}_{s(s)}
\end{aligned}
$$

Après avoir exprimé les grandeurs rotoriques à travers leurs actions sur le stator, et appliqué la rotation d'un angle $\theta_{s}$, on obtient le modèle dans le référentiel (T)lié au champ tournant :

$$
\begin{aligned}
& \bar{V}_{s(T)}=R_{s} \bar{I}_{s(T)}+\frac{d \bar{\Phi}_{s(T)}}{d t}+j \frac{d \theta_{s}}{d t} \bar{\Phi}_{s(T)} \\
& \bar{V}_{r(T)}=R_{r} \bar{I}_{r(T)}+\frac{d \bar{\Phi}_{r(T)}}{d t}+j \frac{d \theta_{r}}{d t} \bar{\Phi}_{r(T)} \\
& \bar{\Phi}_{s(T)}=L_{s} \bar{I}_{s(T)}+L_{s r} \bar{I}_{r(T)} \\
& \bar{\Phi}_{r(T)}=L_{r} \bar{I}_{r(T)}+L_{s r} \bar{I}_{s(T)}
\end{aligned}
$$




\section{2-2. Couple électromagnétique}

A partir de l'expression de la puissance instantanée, on peut distinguer la valeur de la puissance électromagnétique qui est responsable du couple :

$$
P_{e m}=\omega_{s}\left(\Phi_{s d} I_{s q}-\Phi_{s q} I_{s d}\right)
$$

Avec :

$$
\omega_{s}=p_{0} \Omega_{s}
$$

Où $p_{0}$ est le nombre de paires de pôles, et on obtient :

$$
T_{e m}=p_{0}\left(\Phi_{s d} I_{s q}-\Phi_{s q} I_{s d}\right)
$$

\section{2-3. Simulation du fonctionnement du moteur}

Les paramètres de construction du moteur considéré sont les suivants :

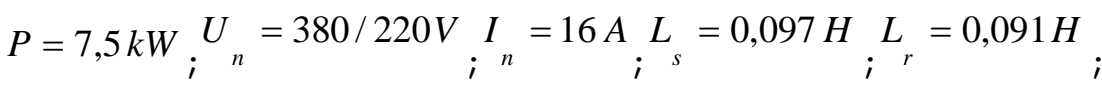

$$
\begin{aligned}
& L_{s r}=0,091 H R_{s}=0,63 \Omega R_{r}=0,4 \Omega{ }_{i}{ }_{t}=0,22 \mathrm{~kg} \cdot \mathrm{m}^{2}{ }_{;} p_{0}=2 .
\end{aligned}
$$

\section{2-3-1. Commande en courant}

Le moteur étant alimenté à l'aide d'un onduleur de courant, on considère qu'un système de trois courants triphasés sera ॥ injecté $॥$ dans les enroulements du stator [4].

Les expressions (6), (7), et (8) donnent l'équation d'état suivant :

$$
\left[\begin{array}{c}
\frac{d I_{r d}}{d t} \\
\frac{d I_{r q}}{d t}
\end{array}\right]=\left[\begin{array}{cc}
-\frac{1}{\tau_{r}} & \omega_{r} \\
-\omega_{r} & -\frac{1}{\tau_{r}}
\end{array}\right] \cdot\left[\begin{array}{c}
I_{r d} \\
I_{r q}
\end{array}\right]+\left[\begin{array}{cc}
0 & \frac{L_{s r}}{L_{r}}\left(\omega_{s}+\omega_{r}\right) \\
-\frac{L_{s r}}{L_{r}}\left(\omega_{s}+\omega_{r}\right) & 0
\end{array}\right] \cdot\left[\begin{array}{l}
I_{s d} \\
I \\
s q
\end{array}\right]
$$




\section{2-3-2. Commande en tension}

Le moteur est alimenté par un onduleur de tension. Il est nécessaire de former, en se servant des équations (5), (6), (7) et (8), une équation d'état dont le modèle est du type :

$\left[\frac{d}{d t} I\right]=\left[A_{m}\right] \cdot[I]+\left[B_{m}\right] \cdot[V]$

Avec:

- La matrice d'évolution d'état du moteur :

$\left[A_{m}\right]=\left[\begin{array}{cccc}-\frac{1}{\sigma \tau_{s}} & \frac{\omega_{s}-\omega_{r}}{\sigma}+\omega_{r} & \frac{L_{s r}}{\sigma \tau_{r} L_{s}} & \frac{L_{s r}}{\sigma L_{s}}\left(\omega_{s}-\omega_{r}\right) \\ \frac{\omega_{r}-\omega_{s}}{\sigma}-\omega_{r} & \frac{1}{\sigma \tau_{s}} & \frac{L_{s r}}{\sigma L_{s}}\left(\omega_{r}-\omega_{s}\right) & \frac{L_{s r}}{\sigma \tau_{r} L_{s}} \\ \frac{L_{s r}}{\sigma \tau_{s} L_{r}} & \frac{L_{s r}}{\sigma L_{r}}\left(\omega_{r}-\omega_{s}\right) & \frac{1}{\sigma \tau_{r}} & \frac{\omega_{r}-\omega_{s}}{\sigma}+\omega_{s} \\ \frac{L_{s r}\left(\omega_{s}-\omega_{r}\right)}{\sigma L_{r}} & \frac{L_{s r}}{\sigma \tau_{s} L_{r}} & \frac{\omega_{s}-\omega_{r}}{\sigma}-\omega_{s} & -\frac{1}{\sigma \tau_{r}}\end{array}\right]$

- La matrice de commande du moteur

$$
\left[B_{m}\right]=\left[\begin{array}{cccc}
\frac{1}{\sigma L_{s}} & 0 & -\frac{L_{s r}}{\sigma L_{s} L_{r}} & 0 \\
0 & \frac{1}{\sigma L_{s}} & 0 & -\frac{L_{s r}}{\sigma L_{s} L_{r}} \\
-\frac{L_{s r}}{\sigma L_{s} L_{r}} & 0 & \frac{1}{\sigma L_{r}} & 0 \\
0 & -\frac{L_{s r}}{\sigma L_{s} L_{r}} & 0 & \frac{1}{\sigma L_{r}}
\end{array}\right]
$$




\section{Résultats}

\section{3-1. Résultats de simulation de la commande en courant}

En régime de "basse vitesse I, avec $f_{s 1}=40 \mathrm{~Hz}$ (courbes bleves claires), $f_{s 2}=30 \mathrm{~Hz}$ (courbes rouges), $f_{s 3}=20 \mathrm{~Hz}$ (courbes bleues):

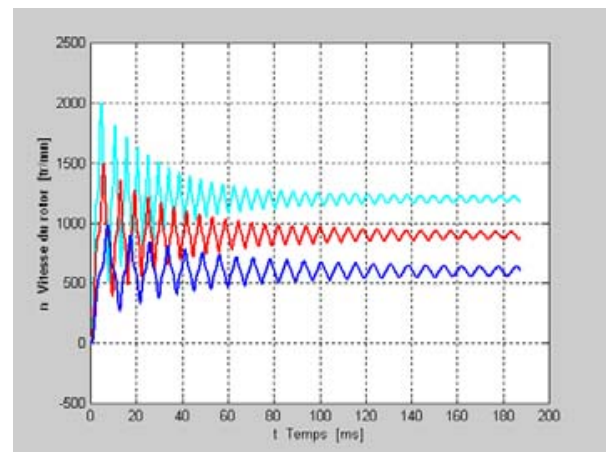

Figure 2 : Vitesse de rotation en fonction du temps

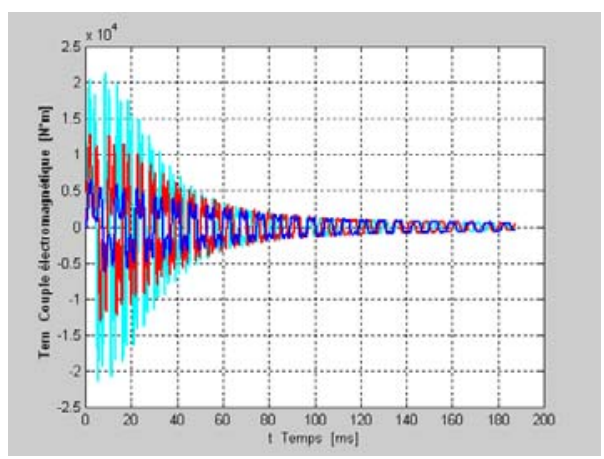

Figure 3 : Couple électromagnétique en fonction du temps

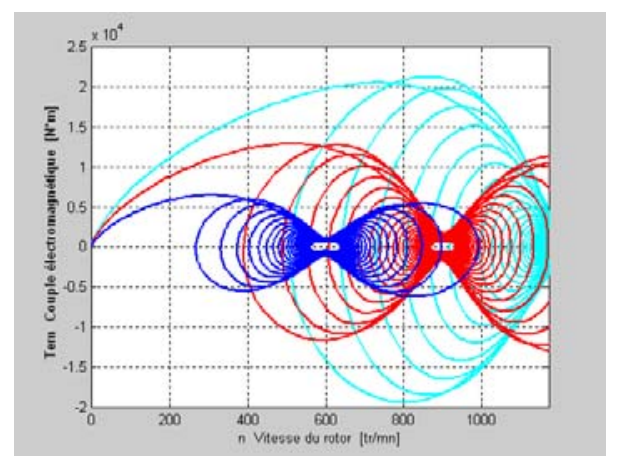

Figure 4 : Couple électromagnétique en fonction de la vitesse

En régime de II survitesse ou défluxage II avec $f_{s 4}=50 \mathrm{~Hz}$ (courbes vertes), $f_{s 5}=75 \mathrm{~Hz}$ (courbes magenta), $f_{s 6}=100 \mathrm{~Hz}$ (courbes noires) et $U_{n}=220 \mathrm{~V}=$ Cte : 


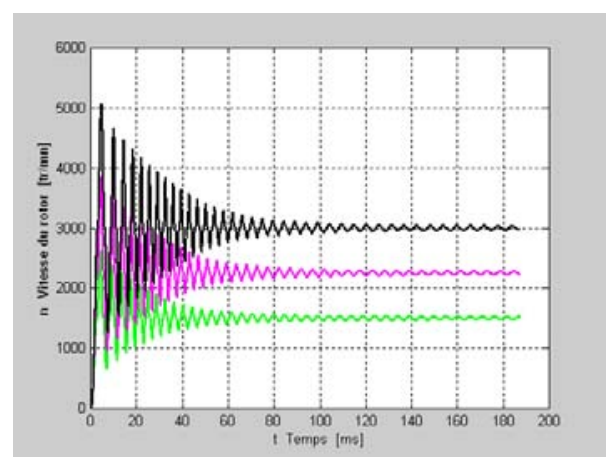

Figure 5 : Vitesse de rotation en fonction du temps

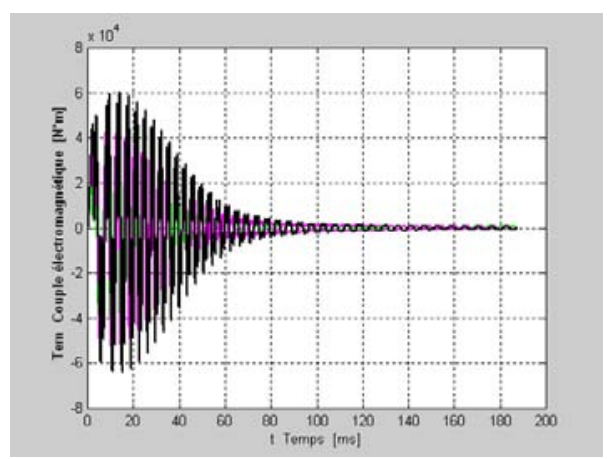

Figure 6 : Couple électromagnétique en fonction du temps

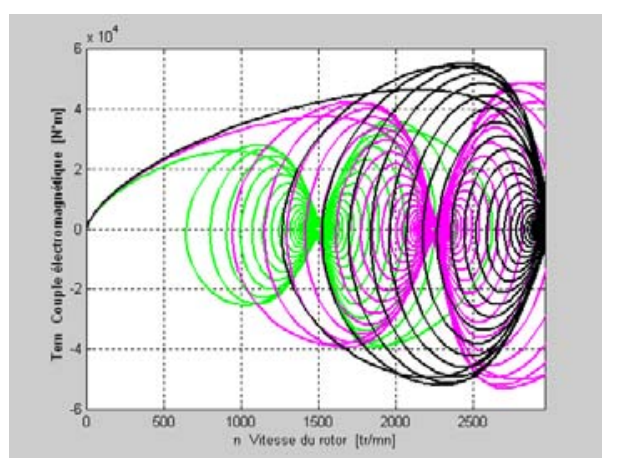

Figure 7 : Couple électromagnétique

en fonction de la vitesse

\section{3-2. Résultats de simulation de la commande en tension}

En régime de ॥ basse vitesse II on aura avec $f_{s 1}=40 \mathrm{~Hz}$ (courbes bleues claires), $f_{s 2}=30 \mathrm{~Hz} \quad$ (courbes rouges), $f_{s 3}=20 \mathrm{~Hz}$ (courbes bleves) et $\Phi_{s}=0,99 \mathrm{~Wb}=\mathrm{Cte}$ : 


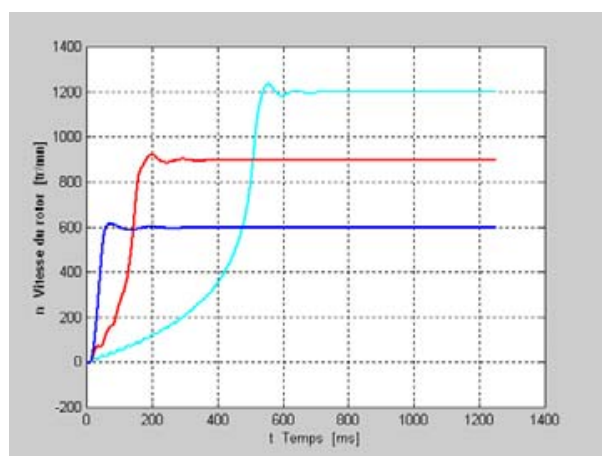

Figure 8 : Vitesse de rotation en fonction du temps

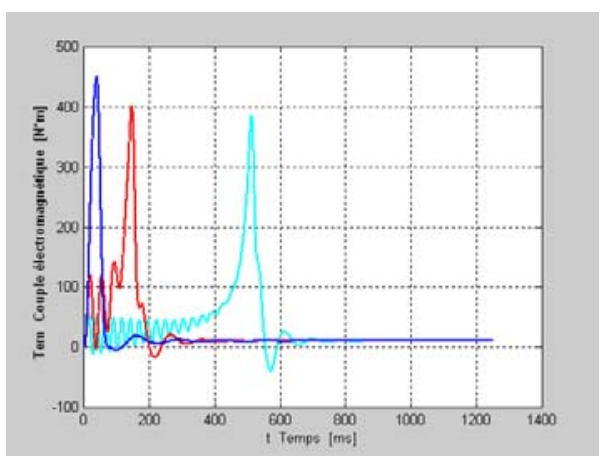

Figure 10 : Couple électromagnétique en fonction du temps

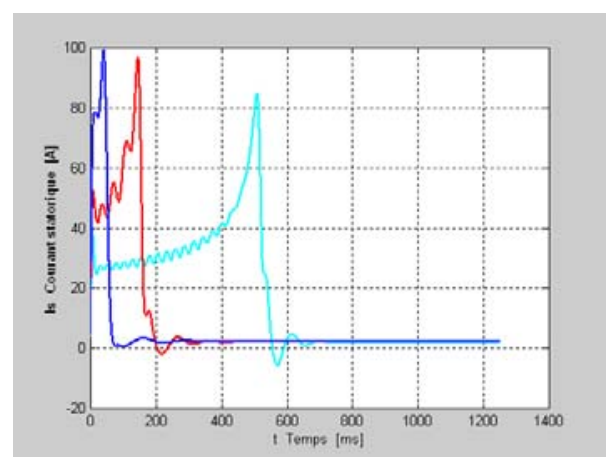

Figure 9 : Courant statorique en fonction du temps

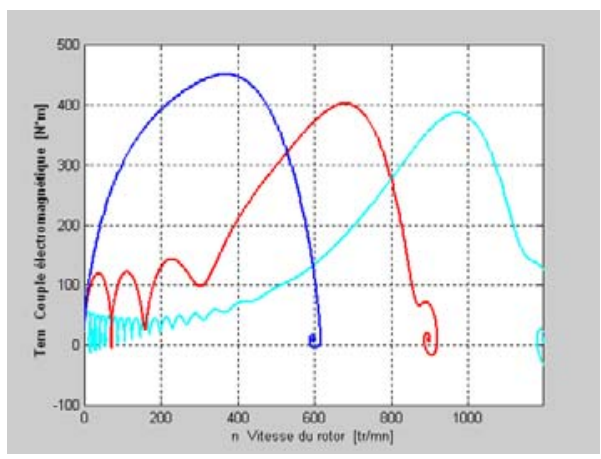

Figure 11 : Couple électromagnétique en fonction de la vitesse

En régime de II défluxage II avec $f_{s 4}=50 \mathrm{~Hz}$ (courbes vertes), $f_{s 5}=75 \mathrm{~Hz}$ (courbes magenta), $f_{s 6}=100 \mathrm{~Hz}$ (courbes noires) et $U_{n}=220 \mathrm{~V}=$ Cte : 


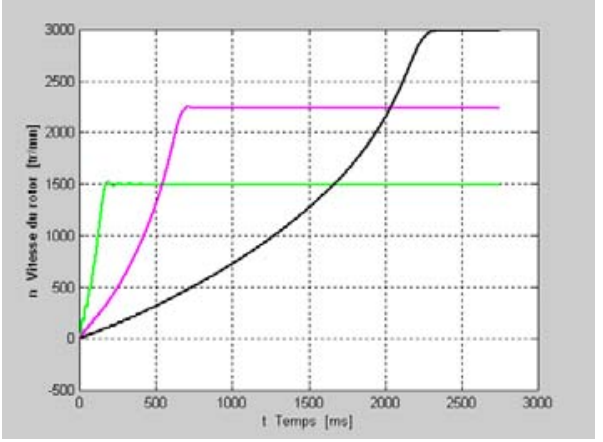

Figure 12 : Vitesse de rotation en fonction du temps

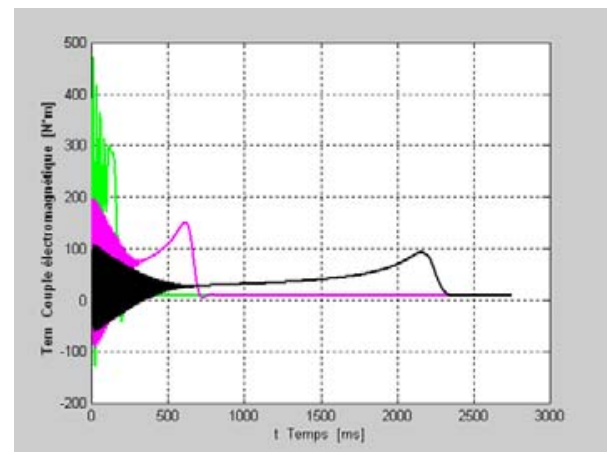

Figure 14 : Couple électromagnétique en fonction du temps

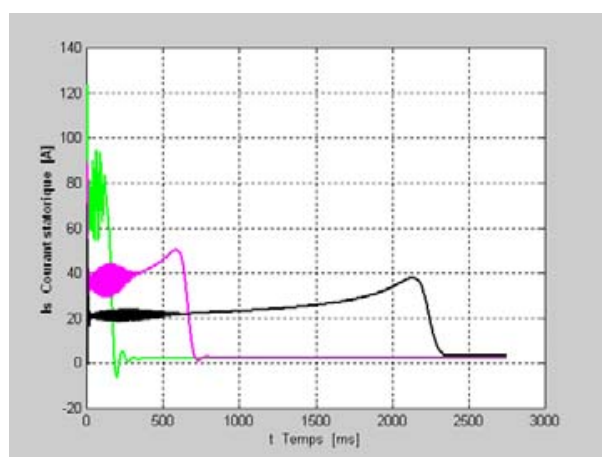

Figure 13 : Courant statorique en fonction du temps

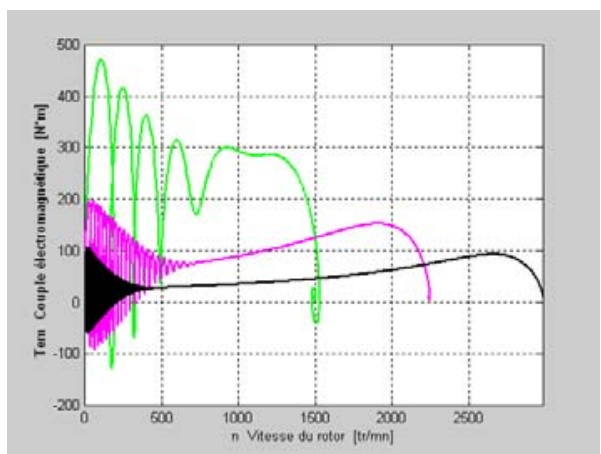

Figure 15 : Couple électromagnétique en fonction de la vitesse

3-3. Analyse du système multivariable

\section{3-3-1. Matrice système du processus}

Le modèle d'état du système est représenté par les équations matricielles suivantes :

$$
\begin{aligned}
& {\left[\frac{d}{d t} I\right]=[A] \cdot[I]+[B] \cdot[V]} \\
& {[\Phi]=[C] \cdot[I]+[D] \cdot[V]}
\end{aligned}
$$

L'illustration de cette modélisation est donnée sur la Figure $\mathbf{1 6 .}$ 


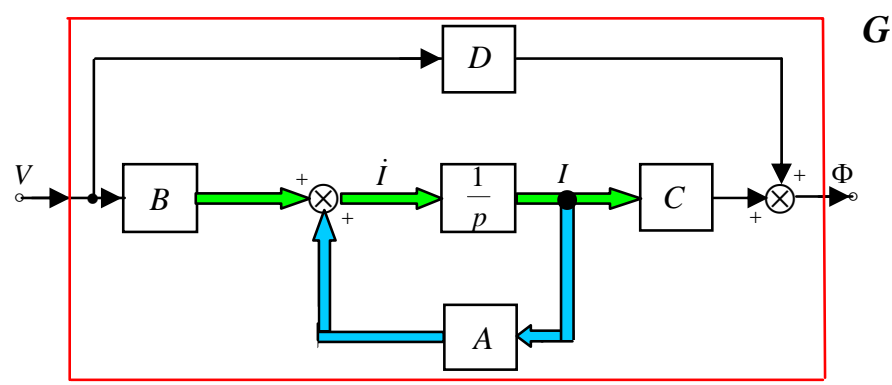

Figure 16 : Schéma fonctionnel du système linéaire dans l'espace d'état

Le redresseur utilisé est un pont de diodes parallèle double triphasé (pont de Graetz) dont la relation entre la tension d'entrée et celle de sortie est :

$U_{d}=1,35 \cdot U$

Où :

$U$ : valeur efficace de la tension du réseau d'alimentation

$U$ $d$ : valeur moyenne de la tension redressée.

La fonction de transfert du filtre LC :

$$
F_{f}(p)=\frac{1}{1+R C p+L C p^{2}}
$$

Avec:

$R$ : résistance de la self

$L$ : inductance de la self

C: capacité du condensateur de filtrage.

L'onduleur est caractérisé par sa matrice de connexion, déterminée avec son modèle mathématique.

La fonction de transfert de l'onduleur sera donc:

$$
F_{o}(p)=r \cdot \frac{U_{c}}{2} \cdot \sqrt{\frac{3}{2}} \cdot \frac{p^{2}}{p^{2}+\pi^{2} \cdot 10^{4}}
$$

Où :

$r$ : coefficient de réglage de l'onduleur vectoriel

$U_{c}$ : tension redressée et filtrée à l'entrée de l'onduleur. 
La simulation du fonctionnement en régime dynamique, avec la fréquence d'alimentation égale à $50 \mathrm{~Hz}$, donne la représentation d' état du moteur dont le traitement sous MATLAB permet d'obtenir la matrice système $G_{m}(p)$.

D'après la chaîne de puissance, la matrice système du processus est donc :

$$
G(p)=G_{m}(p) \cdot G_{o}(p) \cdot G_{f}(p) \cdot G_{r}(p)
$$

Avec:

$$
\begin{aligned}
& G_{m}(p) \text { : matrice système du moteur asynchrone } \\
& G_{o}(p) \text { : matrice système de l'onduleur } \\
& G_{f}(p) \text { : matrice système du filtre } \\
& G_{r}(p) \text { : matrice système du redresseur. }
\end{aligned}
$$

Notons que la matrice système de l'onduleur, celle du filtre ainsi que celle du redresseur sont obtenues à partir de chaque fonction de transfert correspondante à l'aide du logiciel MATLAB.

\section{3-3-2. Courbe des valeurs singulières}

Considérons un système linéaire ayant pour matrice de transfert $[F(p)]$, de rang $k_{\text {, }}$ à $m$ lignes et $n$ colonnes. Les valeurs singulières de la matrice de transfert $\left[F\left(j \omega_{s}\right) \mid\right.$ de rang $k_{r}$ notées $\sigma_{i}(F)$, sont les racines carrées des valeurs propres de $\left[F\left(j \omega_{s}\right)\right] \cdot\left[F\left(j \omega_{s}\right)\right]_{\text {et }}^{T}\left[F\left(j \omega_{s}\right)\right] \cdot\left[F\left(j \omega_{s}\right)\right]$ :

$\left.\sigma_{i}(F)=\sqrt{\lambda_{i}\left\{\left[F\left(j \omega_{s}\right)\right] \cdot\left[F\left(j \omega_{s}\right)\right] T\right.}\right\}=\sqrt{\lambda_{i}\left\{\left[F\left(j \omega_{s}\right)\right] T \cdot\left[F\left(j \omega_{s}\right)\right]\right\}}$

Où :

$$
\begin{gathered}
\lambda_{i}: \text { la } i^{\text {ème }} \text { valeur propre du produit matriciel }\left[F\left(j \omega_{s}\right)\right] \cdot\left[F\left(j \omega_{s}\right)\right]_{\text {ou }}^{T} \\
{\left[F\left(j \omega_{s}\right)\right]^{T} \cdot\left[F\left(j \omega_{s}\right)\right]}
\end{gathered}
$$


$\sigma_{i}:$ la ì ìme valeur singulière de $\left\lfloor F\left(j \omega_{s}\right)\right\rfloor$.

La courbe des valeurs singulières du système en fonction de ${ }^{\omega}{ }_{s}$ est représentée sur la Figure 17.

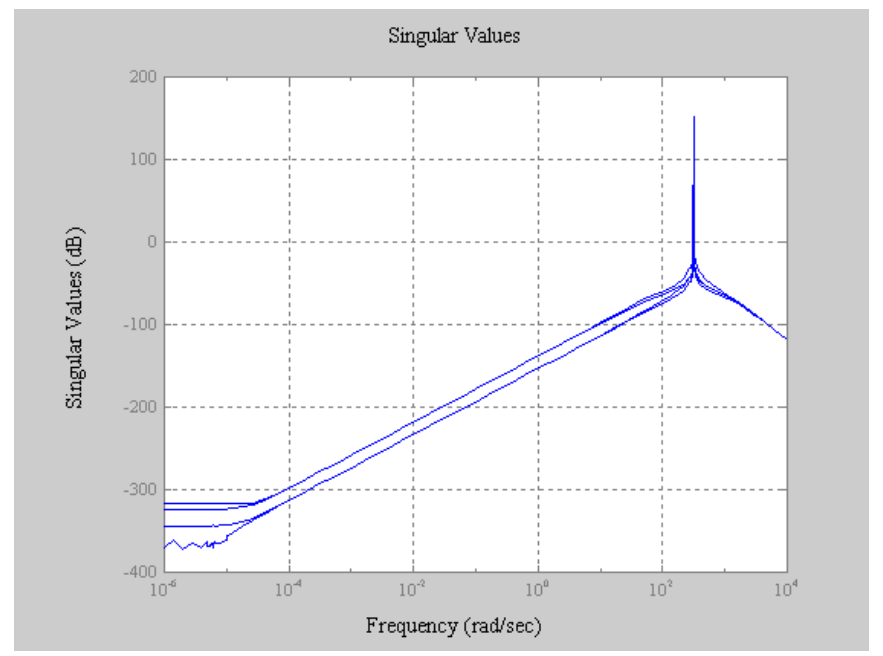

Figure 17 : Courbe des valeurs singulières du système

\section{3-3-2. Courbe de gain}

Les produits matriciels $\left[F\left(j \omega_{s}\right)\right] \cdot\left[F\left(j \omega_{s}\right)\right]^{T}$ ef $\left[F\left(j \omega_{s}\right)\right]^{T} \cdot\left[F\left(j \omega_{s}\right)\right]$ sont des matrices hermitiennes semi définies positives. Ils ont $k$ valeurs propres égales et les éventuelles valeurs propres supplémentaires sont nulles.

Les valeurs singulières ${ }^{\sigma_{i}}(F)$ constituent donc une généralisation, aux systèmes multivariables, de la notion de gain et peuvent être représentés dans le plan de Bode.

La courbe de gain du système en fonction de $\omega_{s}$ est donné sur la Figure 18. 


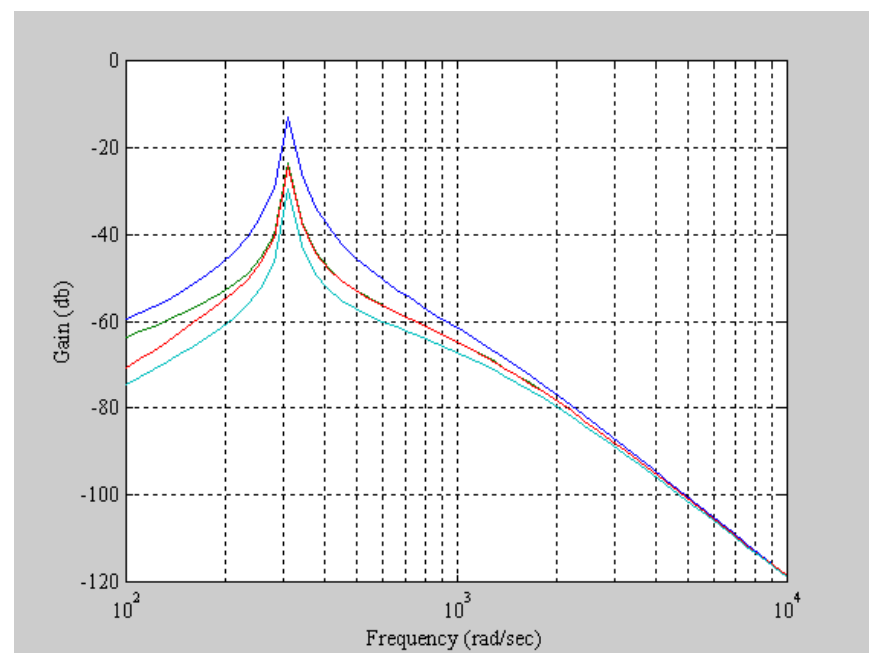

Figure 18 : Courbe de gain du système

\section{3-4. Synthèse du système multivariable}

\section{3-4-1. Modèle du système linéaire avec incertitudes non structurées}

En pratique, parfois la modélisation établie n'est pas suffisamment précise pour traduire fidèlement le comportement d'un système. Souvent les concepteurs ont recours aux modèles avec incertitudes non structurées qui affectent généralement les dynamiques mal connues ou volontairement négligées. L'analyse de la robustesse est effectuée en incorporant toutes les incertitudes de modèle dans une seule matrice de transfert sans lui imposer de structure particulière.

Dans cette étude, en supposant que plusieurs sources d'incertitudes de natures différentes coexistent, on adopté un système avec erreurs de modèle de forme additive directe dont le schéma bloc est donné sur la Figure 19. Le processus perturbé est représenté par $\tilde{G}$, la matrice de la robustesse en stabilité du système par $M_{r s}$ et les erreurs de modélisation ramenées en sortie sont réparties sur le processus $G$. 


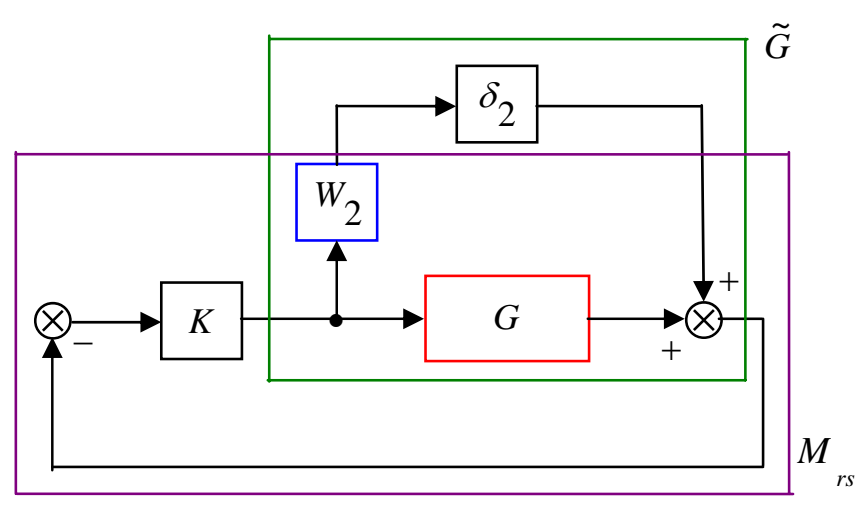

Figure 19 : Système avec erreurs de modèle de forme additive directe

Le régulateur proportionnel a pour fonction de transfert :

$K(p)=1$

La fonction de pondération est un filtre du premier ordre ayant pour expression :

$W_{2}(p)=\frac{0,35 p+1}{0,4 p+500}$

Et on obtient la courbe suivante pour la commande robuste du système :

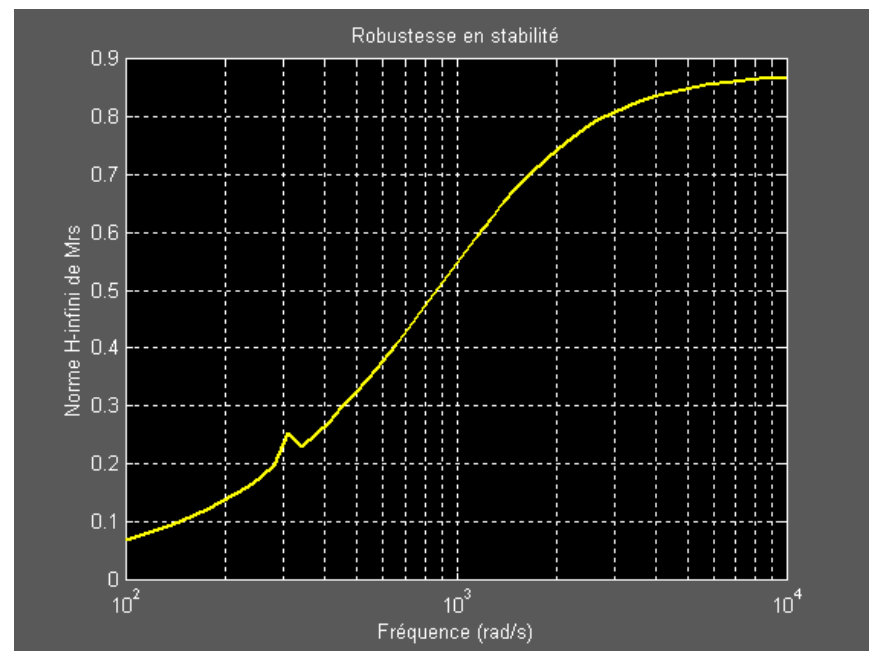

Figure 20 : Norme H-infini de la matrice de robustesse en stabilité avec erreurs de modèle de forme additive directe 


\section{3-4-2. Analyse de la robustesse du système linéaire}

Le système bouclé satisfait à la robustesse en performance si la condition de performance nominale est vérifiée pour toute une classe de processus de matrices de transfert $\tilde{G}$, différent de $G$ par l'une quelconque des incertitudes de modèle. Le schéma bloc retenu est représenté sur la Figure 21.

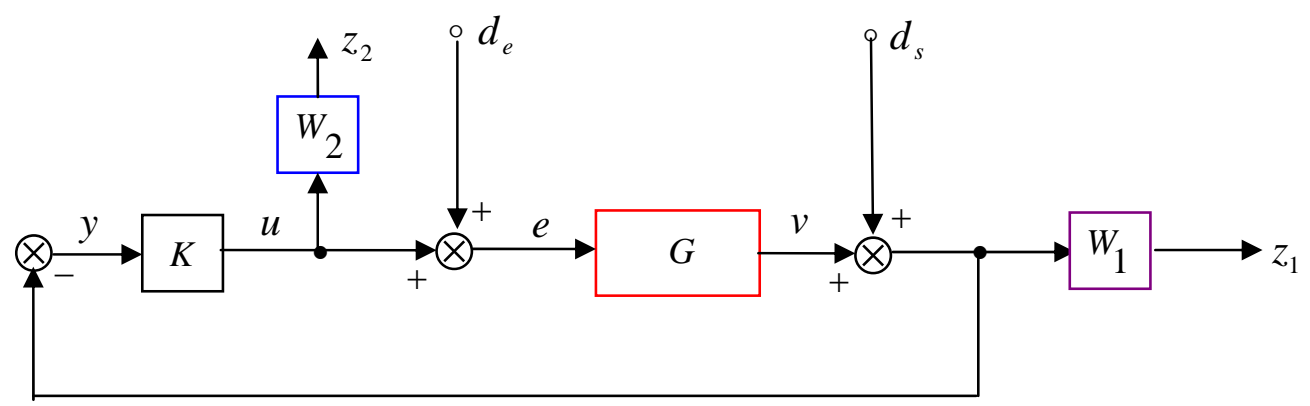

Figure 21 : Schéma bloc du système asservi pour l'analyse de la robustesse

La liste de la notation utilisée :

$y$ : signal d'erreur

$U$ : commande

$e:$ entrée du processus

$v$ : sortie du processus

$d_{e}$ : perturbation à l'entrée du processus

$d_{s}$ : perturbation à la sortie du processus.

Pour atteindre les objectifs du cahier de charges, souvent exprimés dans différents domaines (gabarit fréquentiel, gabarit temporel, critères sur les amortissements de la boucle fermée, critères énergétiques et statistiques...), on introduit des pondérations sur les différents signaux, qui prendront la forme de filtres permettant, suivant le signal auquel elles s'appliquent, de privilégier une plage de fréquences particulière.

Le filtre de pondération ${ }_{1}(p)$, qui possède trois degrés de liberté, a pour expression : 
$W_{1}(p)=\frac{10^{-21}\left(0,55 p+1,5 \cdot 10^{12}\right)}{1,475 p+3 \cdot 10^{-9}}$

Le filtre de pondération ${ }_{2}(p)$ :

$W_{2}(p)=\frac{0,35 p+1}{0,4 p+500}$

En isolant le correcteur $K$, on obtient le schéma bloc contenant la matrice de l'interconnexion en boucle ouverte $P$ et la matrice de transfert en boucle fermée $M$ :

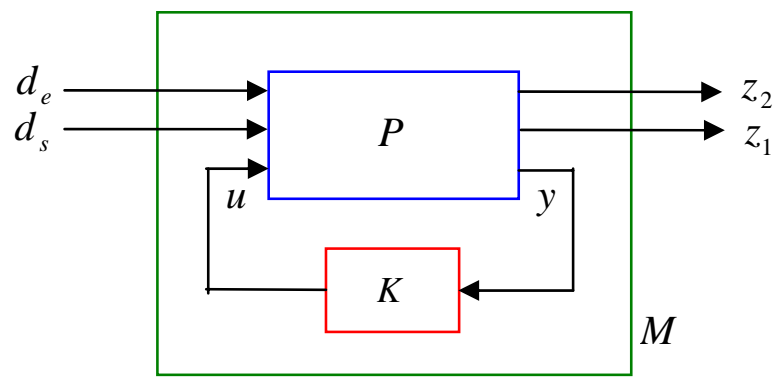

Figure 22 : Schéma bloc transformé du système linéaire

Avec:

$\left[\begin{array}{l}z_{2} \\ z_{1}\end{array}\right]=\left[\begin{array}{ll}M_{11} & M_{12} \\ M_{21} & M_{22}\end{array}\right]\left[\begin{array}{l}d_{e} \\ d_{s}\end{array}\right]$

Le système de la Figure $\mathbf{2 3}$ satisfait la condition de robustesse en stabilité pour toute matrice d'incertitude, appliquée à la partie supérieure de $M_{1}{ }_{u}$ telle que $\left\|\Delta_{u}\right\|_{\infty} \leq 1$ si et seulement si $\forall \omega_{s} \in R, \mu_{\Delta_{u}}\left(M_{11}\right)<1$ ou encore $\left\|M_{11}\right\|_{\infty}<1$

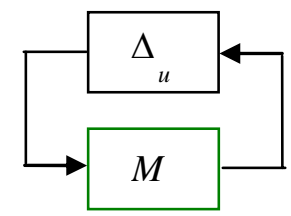

Figure 23 : Schéma général d'étude de la robustesse en stabilité 


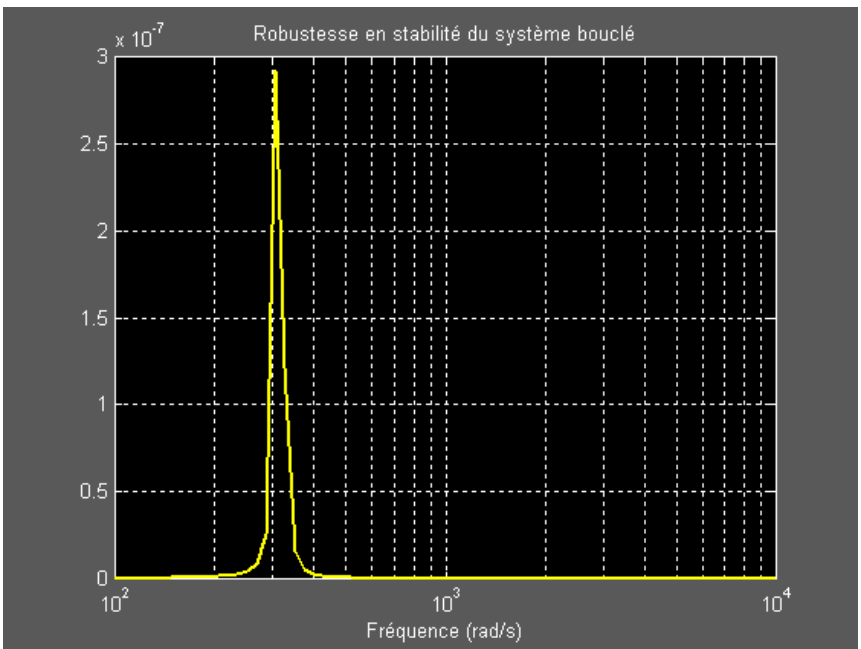

Figure 24 : Robustesse en stabilité du système bouclé

La performance nominale de la Figure 25 est assurée si et seulement si :

$\forall \omega_{s} \in R, \mu_{\Delta_{l}}\left(M_{22}\right)<1 \quad$ ou encore $\quad\left\|M_{22}\right\|_{\infty}<1 ; \Delta_{l}$ étant la matrice d'incertitude du modèle appliquée à la partie inférieure de $M$.

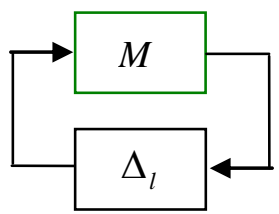

Figure 25 : Schéma général d'étude de la performance nominale

Le système de la Figure 27 satisfait la condition de robustesse en performance pour toute matrice

$\Delta_{r p}=\left\{\operatorname{diag}\left[\Delta, \Delta_{f}\right]\right\}$ avec $\|\Delta\| \leq 1$ si et seulement si $\forall \omega_{s} \in R, \mu_{\Delta}(M)<1$

Avec:

$\Delta$ : matrice d'erreur ou incertitude du modèle

${ }^{\Delta}{ }_{f}$ : matrice d'incertitude fictive pour l'analyse de la robustesse en performance 


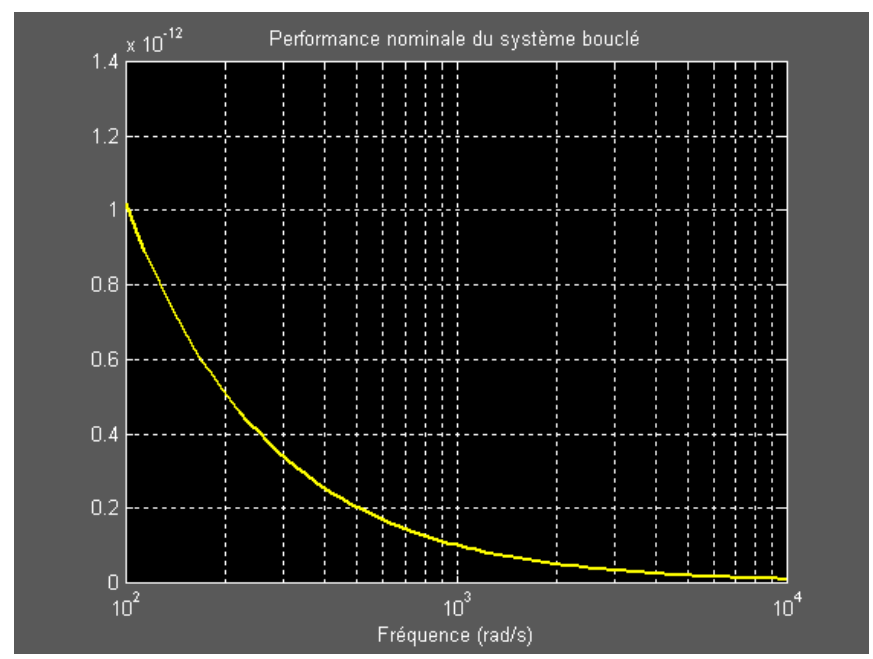

Figure 26 : Performance nominale du système bouclé

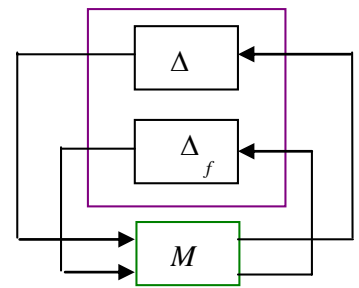

$\Delta_{\text {r p }}$

Figure 27 : Schéma général d'étude de la robustesse en performance

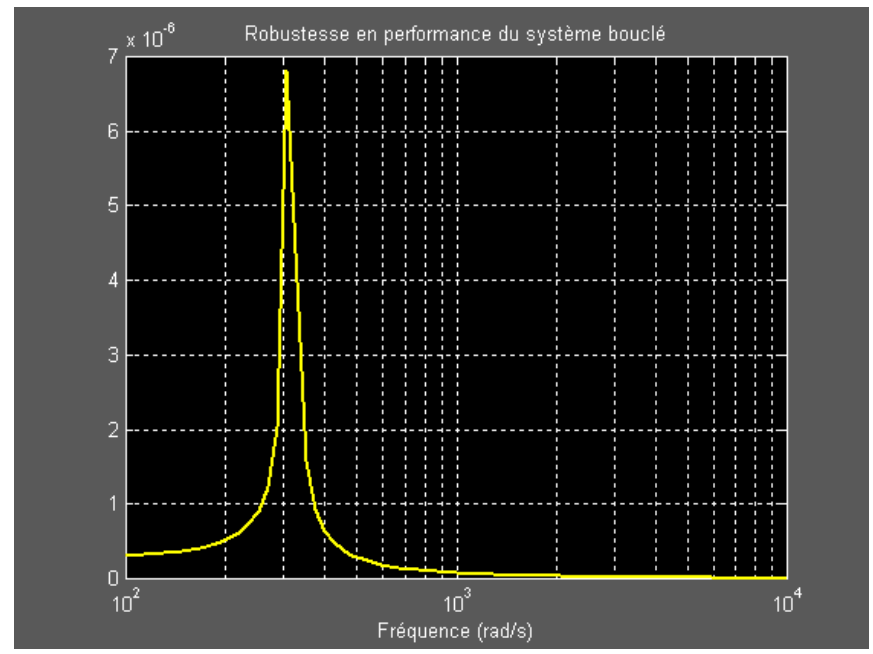

Figure 28 : Robustesse en performance du système bouclé 


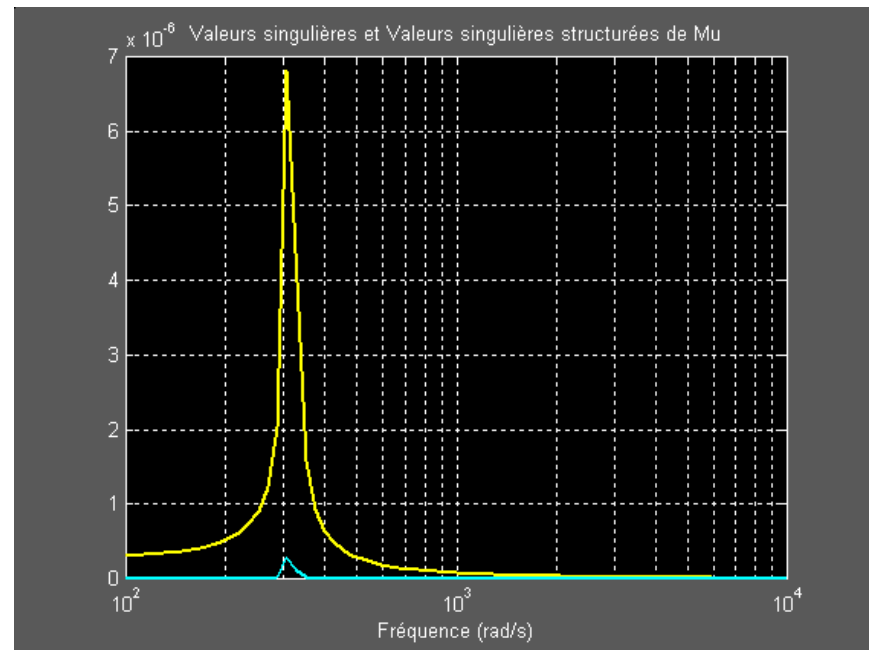

Figure 29 : Valeurs singulières et valeurs singulières structurées de Mu

\section{Discussion}

\section{4-1. Commande en courant}

- Les résultats de simulation en régime dynamique montrent que, pour l'alimentation par onduleur de courant, les caractéristiques du couple et du courant en fonction du temps sont pourvues d'oscillations non amorties.

- Les caractéristiques du couple en fonction de la vitesse auraient dô se terminer au point de fonctionnement, mais à cause des ondulations de la vitesse et du couple, elles prennent fin en formant une petite boucle.

- En agissant directement sur la fréquence d'alimentation des enroulements statoriques, le fonctionnement du moteur peut donc être affecté de réponses oscillatoires peu amorties ou d'instabilités intempestives.

- L'alimentation par onduleur de tension s'avère alors être le plus adapté pour les moteurs asynchrones à rotor à cage de faible ou moyenne puissance.

- L'alimentation par onduleur de courant est plutôt réservée aux installations de grande puissance nécessitant une importante récupération d'énergie. 


\section{4-2. Système multivariable}

- La courbe des valeurs singulières présente un pic trop élevé au niveau de la fréquence nominale $f_{s}=50 \mathrm{~Hz}\left(\omega_{s}=314,16 \mathrm{rd} / \mathrm{s}\right)$.

- La norme ${ }_{\infty}$, qui est la norme des matrices de transfert rationnelles à coefficients réels correspondant à un système stable, est trop grande.

- Le système nécessite donc d'être corrigé.

\section{Conclusion}

- La valeur de la norme ${ }^{H}$ de la matrice de transfert Mu est égale à 0,7766 pour la pulsation statorique du moteur $\omega_{s}=314,1592 \mathrm{rad} / \mathrm{s}$.

- Le système est robuste en stabilité.

- Le système bouclé perturbé satisfait la robustesse en performance.

\section{Références}

[1] - J. P. LOUIS, II Modélisation des machines électriques en vue de leur commande II, Ed. Hermès

[2] - C. CANUDAS DE WIT, " Modélisation, commande vectorielle et DTC ॥, Ed.Hermès, Paris, (2000)

[3] - M. MOKHTARI, "MATLAB 5.2 - 5.3 et SIMULINK“, Ed. Springer, Verlag, Berlin, Heidelberg, (2000)

[4] - L. MUTREL, II Le moteur asynchrone II, Ed. Ellipses, Paris, (1999)

[5] - L. JAULIN, II Représentation d'état pour la modélisation et la commande des systèmes II, Ed. Hermès, Paris, (2005)

[6] - H. ABOU-KANDIL, I La commande optimale des systèmes dynamiquesil, Ed. Hermès, Paris, (2004)

[7] - C. Y. VIBET, II Systèmes asservis linéaires continus II, Ed. Ellipses, Paris, (1987)

[8] - M. VILLAIN, "Signaux et systèmes continus et échantillonnés II, Ed. Ellipses, Paris, (1996) 
[9] - M. VILLAIN, II Systèmes asservis linéaires ॥, Ed. Ellipses, Paris, (1996)

[10] - J. F. MASSIEU, P. DORLEANS, II Modélisation et analyse des systèmes linéaires II, Ed. Ellipses, Paris, (1996)

[11] - B. LEMAIRE-SEMAIL, F. BOUILLAUT et A. RAZEK, II Modélisation et commande d'un moteur asynchrone saturé avec contrôle dynamique du flux II, Journal de Physique III, France 1 (1991) 1509-1528

[12] - J. L. LIN, I. POSTLETHWAITE and D. W. GU, " $\mu_{-K}$ Iteration : A New Algorithm for

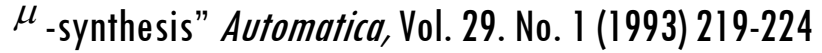

[13] - T. OURTH, "Commande vectorielle d'un moteur asynchrone sans capteur", Journal de Physique III, France 3 (1993) 1123-1133

[14] - [Y. A. CHAPUIS, D. ROYE et S. COURTINE, II Commande directe du couple d'une machine asynchrone par le contrôle direct de son flux statorique II, Journal de Physique III, France 5 (1995) 863-880

[15] - J. C. PAPAZIAN, J. P. ROGNON, D. ROYE et Ph. DELBOSC, II Comparaison entre les différentes commandes d'un moteur asynchrone pour un véhicule électrique en vue de minimiser les pertes de l'onduleur II, The European Physical Journal Applied Physics, 2 (1998) 63-77 